\title{
VALORACIÓN CONTINGENTE DEL SERVICIO DE AGUA POTABLE EN LA YARADA - LOS PALOS TACNA 2016
}

\author{
CONTINGENT VALUATION OF THE DRINKING WATER SERVICE IN LA \\ YARADA - LOS PALOS TACNA 2016
}

${ }^{1}$ Everth Castro y Céspedes, ${ }^{2}$ Nelly Arévalo Solsol

\begin{abstract}
RESUMEN
Actualmente la irrigación La Yarada-Los Palos, que abarca el distrito del mismo nombre, no cuenta con el servicio de agua potable; por lo cual debe cubrir esta necesidad tomando el agua de los pozos de explotación agrícola, expuestos a microorganismos perjudiciales y contaminados naturalmente por la presencia de arsénico, boro, níquel y otros elementos. En vista de que el recurso hídrico del acuífero se encuentra en veda por la sobreexplotación agrícola, es importante valorar económicamente este servicio usando el método de la valoración contingente; ya que existe una población mayoritariamente dedicada a la actividad agrícola que requiere contar con este servicio de agua potable. El objetivo de la investigación fue determinar el valor económico que el poblador de La Yarada-Los Palos estaría dispuesto a pagar (DAP), por contar con el servicio de agua potable. Para estimar la DAP, la información de las encuestas fue vaciada en el programa económico GRETL modelo LOGIT. Los resultados indican que los pobladores beneficiarios y no beneficiarios estarían dispuestos a pagar hasta 48,39 soles, además de un pago adicional mensual para la conservación del acuífero del valle Caplina de 2,48 soles, porque valoran este recurso para las generaciones futuras.
\end{abstract}

Palabras clave: Agua potable, disposición a pagar, regresión logística, valoración contingente.

\begin{abstract}
At the moment the irrigation Yarada-Los Palos that includes the district of the same name does not count on the service of drinking water having to take the water of the wells of agricultural operation that are exposed to harmful microorganisms and contaminated naturally by the presence of arsenic, boron, nickel and other elements to cover this necessity. In view of the fact that the water resource of the aquifer is closed due to agricultural overexploitation, it is important to value this service economically using the contingent valuation method since there is a population mainly dedicated to agricultural activity that requires this drinking water service. The objective of the investigation was to determine the economic value that the residents of La Yarada-Los Palos would be willing to pay (DAP) for having drinking water service. To estimate the WTP, the information from the surveys was emptied into the GRETL economic program model LOGIT. The results indicate that the beneficiary and non-beneficiary would be willing to pay up to 48,39 soles and an additional monthly payment for the conservation of the Caplina valley aquifer of 2,48 soles, because they value this resource for future generations.
\end{abstract}

Keywords:Drinking water, willingness to pay, logistic regression, contingent valuation.

\section{INTRODUCCIÓN}

El servicio de agua potable en el distrito La Yarada-Los Palos adquiere un grande interés, debido a que este servicio tiene como fuente de agua el acuífero del valle Caplina, que se encuentra en veda por la sobreexplotación del acuífero, cuya recarga de agua es menor a la extracción; considerando que las aguas subterráneas son un recurso muy valioso en Tacna, por ser una región ubicada en la cabecera del desierto de
Atacama, más aún cuando su volumen viene disminuyendo y deteriorándose. Por lo que se hace necesario investigar el verdadero valor de este importante servicio de agua potable en el distrito La Yarada-Los Palos, para lo cual se usó el método de la valoración contingente a fin de determinar el verdadero valor económico de este servicio así como considerar su valor de uso y valor ambiental para las generaciones futuras, teniendo en cuenta la gestión sostenible y racional del acuífero del valle Caplina.

\footnotetext{
${ }^{1}$ Profesional en Recursos Hídricos de la Administración Local de Agua Caplina Locumba. Tacna-Perú. E-mail: ecastro@ana.gob.pe ${ }^{2}$ Facultad de Ciencias Agropecuarias. Universidad Nacional Jorge Basadre Grohmann. Tacna-Perú. E-mail: arevalonelly@hotmail.com 
Actualmente se tiene referencia de trabajos de investigación realizados sobre valoración contingente, por los servicios ambientales que prestan los ecosistemas y que servirán para evitar su deterioro y conservarlos mediante la gestión sostenida y sustentable de dichos recursos. Así Garzón (2013) refiere que los bienes y servicios ambientales no pueden ser valorados económicamente a través de mercados definidos, por tanto ha sido necesaria la implementación de metodologías como la valoración contingente. En los años sesenta y setenta, la valoración contingente comenzó a adquirir importancia especialmente para la estimación de valores de no uso. Bockor et al. (2005) estudiaron en San Jerónimo (Guatemala) la valoración del agua potable en función del bosque que presta el servicio ambiental. Cabe precisar que la tierra es propiedad de la comunidad y un alto porcentaje de la misma está cubierto por vegetación característica de las zonas de vida de bosque húmedo y pluvial, que contribuyen en la captación del agua como regulador del sistema de agua potable del casco urbano en la localidad. La población mostró una apertura a pagar más en la factura de agua potable, por la reforestación/protección del bosque; mientras que percibe como justo el pago mínimo por el actual servicio de agua (Q.6,00/mes), pues el servicio no brinda la calidad adecuada de agua. No obstante, si mejora el servicio, las personas estarían dispuestas a pagar más.

Asimismo, Herrador y Dimas (2001) estudiaron la valoración económica del agua potable para el Área Metropolitana de San Salvador (AMSS) y señalaron que los resultados están relacionados al valor de uso indirecto que los bosques y agroecosistemas de la parte alta de la cuenca proporcionan a las familias del área metropolitana, a través del suministro de agua proveniente del río Lempa. Concluyeron que un $42 \%$ de las respuestas ante la pregunta de la Disposición a pagar (DAP) para conservar los bosques resultaron ser negativas, a pesar de que un $78 \%$ de los entrevistados considera que la presencia de cobertura vegetal es importante en la protección del recurso hídrico; lo que pone de manifiesto que la apropiación del concepto "servicios ambientales" y el de "pago" por los mismos es aún incipiente. Brunett et al.(2010) estudiaron la disposición a pagar, por parte de los usuarios del Valle de Toluca para conservar el Parque Nacional Nevado de Toluca (México), que recarga el acuífero Toluca en el marco de un programa de Pago por Servicios Ambientales Hidrológicos. Los resultados muestran que los usuarios dispuestos a pagar rebasan el $50 \%$, con cantidades que oscilan entre 30 y 80 pesos mensuales; sin embargo, hay un sector de los encuestados que no estaría dispuesto a contribuir, pero realizaría acciones enfocadas al cuidado del medio ambiente.

Del mismo modo, Martínez y Dimas (2007) determinaron la disponibilidad de pago de las comunidades usuarias de los servicios hidrológicos que proveen la parte media y alta de esta subcuenca. En relación con la valoración contingente se puede decir que el $67 \%$ de los entrevistados respondió afirmativamente a la pregunta de la DAP; aunque a medida que los montos contenidos en la pregunta de DAP aumentaban, la probabilidad de obtener respuestas positivas iba disminuyendo. La DAP de los entrevistados fue de Q26,30 familia/mes (US\$3,46 familia/mes), y la suma de las disposiciones a pagar de los habitantes de un total de Q1 millón/año (US\$132 mil/año). Avilés-Polanco et al. (2010) realizaron la valoración económica del acuífero de $\mathrm{La} \mathrm{Paz}$ (Bolivia), que brinda el servicio de provisión de agua potable a un sector de la ciudad. Para esto se utilizó el método de valoración contingente (MVC). Los resultados revelan, también de manera consistente, que la probabilidad de pagar por conservar el servicio de provisión del acuífero es mayor para hogares que reciben algunas horas de agua y están dispuestos a abonar cuatro pesos más que aquellos hogares que no cuentan con restricción.

En la misma línea, Barrantes y Flores (2013) realizaron un estudio para estimar la disponibilidad a pagar(DAP) por la implementación de un programa de conservación y mejoramiento de pastizales (PCMP) en la región Pasco (Perú), mediante el método de valoración contingente. Para estimar la DAP se aplicó una encuesta preliminar de tipo abierta a 30 pobladores y otra cerrada en formato binario a otros 105. Los resultados de la encuesta preliminar abierta permitieron definir siete vectores de pagos (BID) (S/ 1; $5 ; 10 ; 20 ; 25 ; 30$ y 40 ), a partir de los cuales se aplicó una encuesta cerrada a razón de 15 personas por BD. Los resultados de la encuesta cerrada se vaciaron en el programa NLOGIT 3.0 para su corrida, utilizando una distribución LOGIT, en tres etapas. La primera con todas las variables de la encuesta, la segunda con las variables socioeconómicas y la tercera con el ingreso familiar mensual. Los outputs de las tres etapas produjeron resultados similares en los signos, negativo para BID y positivo para los ingresos familiares. La DAP fue S/ 3,94/familia/mes, y reveló que la región Pasco podría recaudar al año 1,95 millones de soles aproximádamente para la implementación del Programa de Conservación y Mejoramiento de Pastizales (PCMP).

\section{MATERIALES YMÉTODOS}

El estudio fue realizado en el distrito La Yarada-Los Palos, ubicado en la parte baja de la cuenca Caplina en las coordenadas: $18^{\circ} 13^{\prime} 45^{\prime \prime} \mathrm{S}$ y $70^{\circ} 28^{\prime} 37^{\prime \prime} \mathrm{O}$ y en una altitud de $62 \mathrm{msnm}$. La ubicación de la cuenca y del área de estudio se indica en las figuras 1 y 2.

\section{Población y muestra}

\section{Población}

E1 estudio constó de una población de 1233 agricultores (población beneficiaria) que conforman los usuarios de agua subterránea de La Yarada-Los Palos, legalmente reconocidos como beneficiarios directos del servicio de agua potable. Además se 
consideró en la investigación a los agricultores del Bajo Caplina 828, vecinos del distrito La Yarada-Los Palos (población no beneficiaria). Lo que llevó a una población total de estudio de 2061 agricultores.

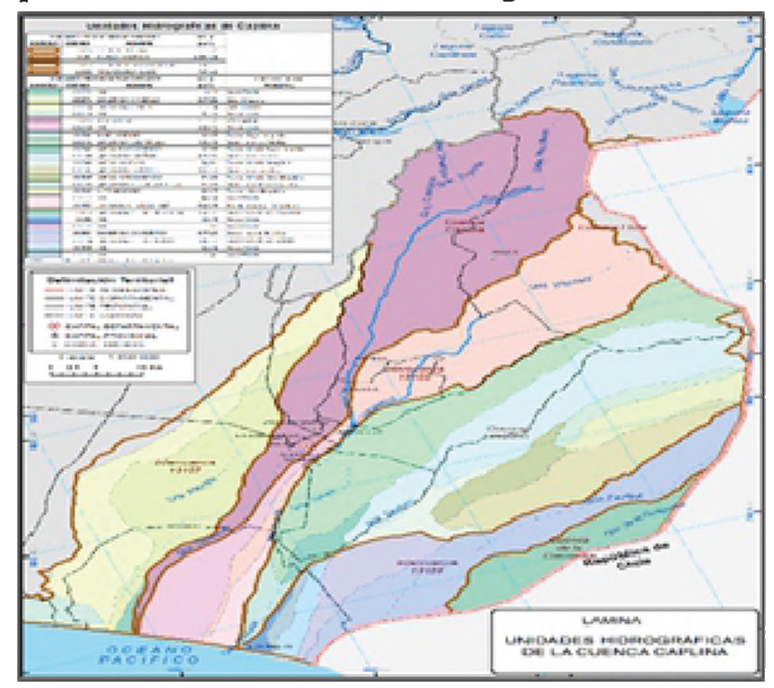

Figura 1. Ubicación de la cuenca Caplina código de UH 13156.

Fuente:Autoridad Nacional del Agua (2013).

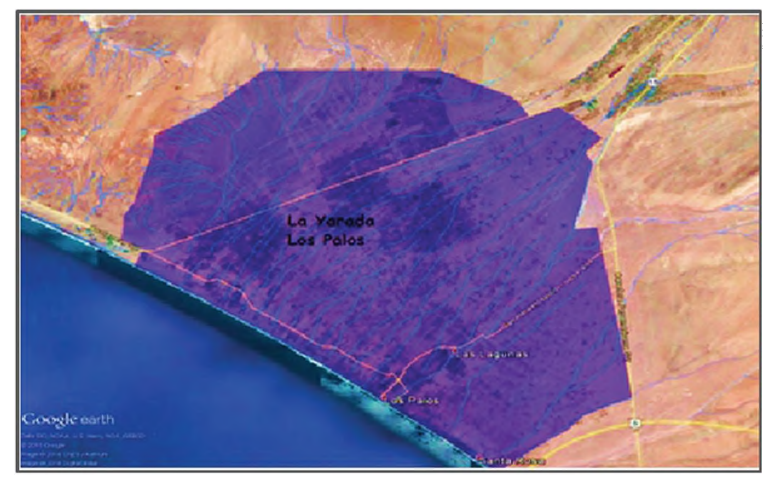

Figura 2. Ubicación del distrito La Yarada Los Palos. Fuente: Google Earth.

\section{Muestra}

El tamafio de la muestra fue calculado de acuerdo al muestreo aleatorio al azar haciendo una muestra de 96 agricultores. La fórmula utilizada para hallar el tamaño de la muestra fue:

$$
\mathbf{n}=\frac{N p(1-p)}{N-1 \frac{B^{2}}{z_{\alpha 2}^{2}}}+p(1-p)
$$

con una probabilidad de 0,9544 y una precisión de 0,05 .

\section{Unidad de análisis}

La unidad de análisis fue el usuario que utiliza el agua subterránea del distrito La Yarada-Los Palos.

\section{Vehículo de pago}

El medio de pago fue la tarifa mensual por el consumo de agua potable.

\section{Modelo estadistico}

El modelo de regresión logistica que se plantea es el siguiente:

$\mathrm{DAP}=\beta \mathrm{0}+\beta 1 \times 1+\beta 2 \times 2+\beta 3 \times 3+\beta 4 \times 4+\beta 5 \times 5+\beta 6 \times 6$ $+\beta 7 \times 7+\beta 8 \times 8$

Donde:

DAP=Disposición a pagar (Variable dependiente)

$\beta_{0}=$ Constante

$\beta 1$..... $\beta 8=$ Coeficiente de variable

$\mathrm{x} 1$........x8 8 Variables independientes

$\mathrm{x} 1=$ vectores de pago $(\mathrm{BID})$

$\mathrm{x} 2=\operatorname{sexo}$

$\times 3=$ edad

$\mathrm{x} 4=$ integrantes de familia

$\mathrm{x} 5=$ nivel de educación

$\mathrm{x} 6=$-ingreso

$\mathrm{x} 7=$ acuífero en veda

$x 8=$ levantar veda

Las hipótesis iniciales del modelo de regresión logistica fueron las siguientes:

(1) Las observaciones $\mathrm{Y} 1, \ldots, \mathrm{Yn}$ son independientes.

(2) Cada Yi sigue una distribucion de Bernoulli.

(3) La probabilidad de que Yi sea igual a 1 (probabilidad de que el individuo pertenezca al grupo A) depende de los valores de las variables X1,..., Xk a traves del siguiente modelo:

$$
\operatorname{Pr}(A)=\operatorname{Pr}(Y i=1)=\frac{1}{1+[e]-\beta 0-\beta 1 \times 1 i-\cdots-\beta j i-\ldots-\beta k x k i}
$$

para $\mathrm{i}=1 \ldots . . \mathrm{n}$

Como en todos los modelos de regresión, estimamos los parámetros del modelo, $\beta 0, \ldots \beta \mathrm{j}, \ldots ., \beta \mathrm{k}$, mediante estimadores puntuales e intervalos de confianza, asimismo estuvimos interesados en algún contraste de hipótesis sobre esos parámetros.

Para dar solución al modelo hemos utilizado el programa económico GRETL (Gnu Regression Econometrics and Time-series Library) según Adkins (2014).

\section{Variables del estudio \\ Dependiente}

Disposición a pagar (DAP). Variable dependiente dicotómica que toma el valor de (1) si la repuesta es Sí a la pregunta de Disponibilidad a Pagar, y (0) en caso contrario.

\section{Independiente}

Vectores de pago (BID): Variable independiente cuantitativa discreta, representa el monto de pago. Toma los diferentes valores de la serie de montos seleccionados, los cuales fueron distribuidos proporcionalmente dentro del total de encuestas.

Edad: Variable independiente cuantitativa discreta. Toma el valor de la edad de cada entrevistado. 
Integrantes de familia: Variable independiente cuantitativa discreta. Representa el número de miembros en la familia.

Hectáreas en producción (ha): Variable independiente cuantitativa discreta. Representa el número de hectáreas totales por agricultor.

Ingreso familiar mensual: Variable independiente cuantitativa discreta, representa el nivel de ingreso mensual por agricultor.

Pago conservación acuífero: Variable independiente cuantitativa discreta, representa el monto de pago. Toma los diferentes valores de la serie de montos seleccionados de 1 a 5 soles.

Residencia: Variable independiente cualitativa ordinal, toma el valor de Tacna $=0 \mathrm{La}$ Yarada $=1$, Los Palos $=2$.

Nivel Educativo: Variable independiente cualitativa ordinal, toma el valor de 0 si la persona no posee educación, 1 si posee educación primaria, 2 si posee educación secundaria, 3 si posee educación universitaria, 4 superior no universitario y 5 si tiene educación a nivel de posgrado.

Conservación acuífera: Variable independiente cualitativa nominal, representa la importancia que el acuífero tiene para el entrevistado.

Acceso Agua potable: Variable independiente cualitativa nominal, representa la importancia que el agua potable tiene para el entrevistado.

Técnicas e instrumentos para recolección de datos Para la recolección y procesamiento de la investigación se ha seguido la secuencia según el diagrama.

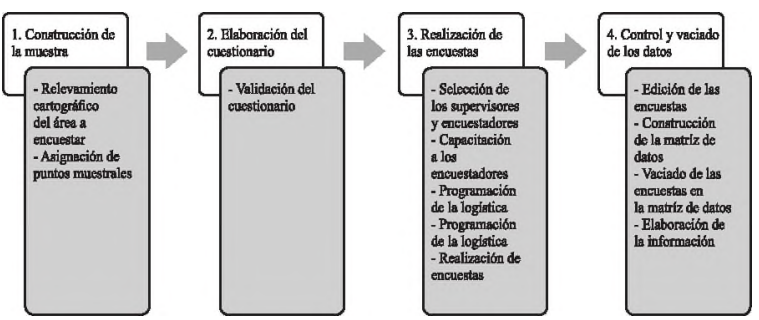

Figura 3. Diagrama secuencial de la metodología usada.

Fuente: Elaboración propia.

Las encuestas fueron realizadas entre los meses de octubre, noviembre y diciembre de 2016 entrevistando mediante muestreo probabilístico a los beneficiarios de La Yarada-Los Palos. Se utilizaron encuestas previamente validadas, mediante la técnica de la respuesta dicotómica o de "referéndum", uno de los enfoques más recomendado para encarar la valoración de un cambio ambiental, utilizando el Método de Valoración Contingente (MVC). En la primera etapa se aplicó una encuesta de carácter abierto, compuesta de 10 preguntas, a 105 personas elegidas al azar sin distinción de sexo y como única restricción de elección que fueran mayores de edad y contribuyan económicamente a la canasta básica familiar. Las primeras nueve preguntas fueron de carácter general y tuvieron que ver con el conocimiento del acuífero del valle Caplina y servicios ambientales; mientras que la décima pregunta fue "¿Cuánto estarían dispuestos a pagar mensualmente como incremento para la conservación del acuífero del río Caplina evitando así la sobreexplotación del acuífero y beneficiándose de sus servicios ambientales?".

Con esta encuesta inicial se pudo medir el nivel de conocimiento del acuífero río Caplina y cómo este influye en su estilo de vida. Se obtuvo así cinco vectores de pagos o $\mathrm{BID}$, que sirvieron posteriormente para diseñar la encuesta cerrada de formato binario (Riera et al., 2005; Fasciolo, 2002).

En una segunda etapa se aplicó una encuesta cerrada de formato binario a 10 usuarios por BID de La YaradaLos Palos (población beneficiaria) y estimado en la primera etapa, haciendo un total de 50 encuestas. Asimismo, se aplicó esta encuesta a los usuarios de Bajo Caplina (población no beneficiaria); lo cual permitió estimar la DAP de los pobladores para el pago por el servicio del agua potable del acuífero valle Caplina con una probabilidad 0,9544 y una precisión del 0,05 en promedio (Chaves, 2008; Villena \& Lafuente, 2012). La encuesta tenía 18 preguntas: siete fueron de carácter social (ingreso familiar mensual, estado civil, edad, sexo, nivel educativo y miembros de familia); nueve de carácter ambiental (conocimiento de la veda del acuífero valle Caplina, acceso al agua potable); y dos de carácter económico (si aceptaban o rechazaban el pago adicional mensual para la implementación de un Programa de Conservación del acuífero La Yarada) (Whitehead, 1990; Riera et al., 2005; Villena \& Lafuente, 2012).

\section{Procesamiento y análisis de datos}

Para el procesamiento de datos se utilizó el software estadístico Gretl (Gnu Regression Econometrics and Time-series Library): un software libre desarrollado en la Universidad de Wake Forest, universidad privada ubicada en Winston-Salem, Carolina del Norte de los EE.UU.

Para estimar la DAP, la información de las encuestas fue vaciadas en el programa económico GRETL, y corrida dos veces. En la primera corrida para la población beneficiaria donde se establecieron las magnitudes de los signos y coeficientes de regresión para cada una de las variables incorporadas al modelo LOGIT. En la segunda corrida para la polación beneficiaria y población no beneficiaria, se estimó al igual que en la primera corrida los coeficientes de regresión, pendientes de los coeficientes y la DAP de cada uno de las variables incorporadas al modelo.En 
todas las corridas se estimó la bondad de ajuste como el $\mathrm{R}^{2}$ Mc Fadden y Chi -Cuadrado, lo que permitió establecer la capacidad de predicción correcta del modelo (Whitehead, 1990; Loomis et al., 2008; Han et al., 2011; Villena \& Lafuente, 2012)

\section{RESULTADOS}

Para la comparación de los resultados se empleó el método de regresión logística, modelo logit recomendado por Fasciolo (2002), del Instituto Nacional del Agua de la Argentina- INA-CELA, por ser más confiable y real para este tipo de estudios, en comparación con otros modelos como el probit y el tobit. Desde el punto de vista matemático, es una función extremadamente flexible y fácil de utilizar y tiene una interpretación relativamente sencilla; y también la evidencia empírica ha demostrado que este modelo es adecuado en la mayoría de los casos en los cuales la respuesta es binaría (Moscote y Arley 2012).

Valor económico por el servicio de agua potable en la población beneficiaria

Tabla 1. Características ambientales y de salud de la población beneficiaria en el distrito La Yarada-Los Palos. Tacna 2016.

\begin{tabular}{|c|c|c|c|}
\hline \multirow{3}{*}{$\mathrm{N}^{*}$} & \multirow{3}{*}{ Pregunta } & \multicolumn{2}{|c|}{ Respuesta } \\
\hline & & & \\
\hline & & sl & $\mathrm{NO}$ \\
\hline 1 & $\begin{array}{l}\text { ¿Es importante para usted consener las aguas del aculfiero del valle } \\
\text { Cap ins? }\end{array}$ & 86,88 & 3,12 \\
\hline 2 & ¿Sabe ustac qua al acu froro del valle Caplina essiá en veda? & 48,00 & 52,00 \\
\hline 3 & $\begin{array}{l}\text { LUsted conoce cua para usar las aguas de La Yaracia sa nocosilie } \\
\text { licencia? }\end{array}$ & 78,20 & 20,80 \\
\hline 4 & ¿En su precia tema azua de su pozo para su alimentación? & 84,40 & 16,60 \\
\hline 5 & ¿Existe dagüü en su precio? & 4.32 & 95,68 \\
\hline 6 & 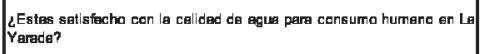 & 35,52 & 64,48 \\
\hline 7 & ¿Debe haber agua potable en el distitto La Yarada Los Palos? & 96,88 & 3,12 \\
\hline 8 & $\begin{array}{l}\text { ¿Considera ustad la falta de acua polatile le ocasicna problemas a su } \\
\text { salud? }\end{array}$ & 82,32 & 17,68 \\
\hline 8 & 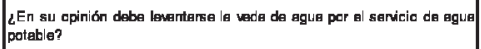 & 72,96 & 27,04 \\
\hline 10 & $\begin{array}{l}\text { ¿Pagerla adicionalmente usted mensualmente por la conseneción del } \\
\text { aculfter la Yarada? }\end{array}$ & 75,00 & 25,00 \\
\hline
\end{tabular}

Fuente: Elaboración propia.

En la tabla 1, se puede observar los resultados de la encuesta abierta en formato binario realizada a los beneficiarios del servicio de agua potable, los cuales señalan que el $96,88 \%$ de los encuestados considera la importancia que significa la conservación del acuífero del valle Caplina, el $79,20 \%$ de los encuestados considera que se debe contar con licencia para usar las aguas; no obstante, únicamente el $48,00 \%$ sabe que el acuífero está en veda.

En cuanto al servicio de agua potable, el $84,40 \%$ toma agua de sus pozos agrícolas para abastecerse de este servicio, el 64,48\% no está satisfecho con la calidad del agua que consumen. Asimismo el 96,88\% opina que debe haber agua potable en La Yarada-Los Palos. El $82,32 \%$ de los encuestados considera que la falta de agua potable le afecta la salud.

Por último, el 72,96\% manifiesta que debe levantarse la veda para contar con el servicio de agua potable. Asimismo, el $75,00 \%$ opina que pagaría adicionalmente para la conservación del acuífero.

Tabla 2. Variables socioeconómicas y ambientales por el servicio de agua potable en la población beneficiaria en el distrito La Yarada-Los Palos. Tacna 2016

\begin{tabular}{|c|c|c|c|c|}
\hline Variables & Media & Máximo & Mínimo & Desviación \\
\cline { 4 - 5 } & 0,34 & 1 & 0 & Estándar \\
\hline Sexo & 44,63 & 80 & 17 & 17,47 \\
\hline $\begin{array}{c}\text { Zona de } \\
\text { residencia }\end{array}$ & 0,62 & 2 & 0 & 0,65 \\
\hline $\begin{array}{c}\text { Integrantes } \\
\text { de familia }\end{array}$ & 4,63 & 10 & 0 & 1,87 \\
\hline $\begin{array}{c}\text { Nivel de } \\
\text { educación }\end{array}$ & 2,53 & 5 & 1 & 0,94 \\
\hline $\begin{array}{c}\text { Ingreso } \\
\text { Acuifero veda }\end{array}$ & 1640,24 & $6,6946,00$ & 0 & 1857,34 \\
\hline $\begin{array}{c}\text { Acceso agua } \\
\text { potable }\end{array}$ & 0,92 & 1 & 0 & 0,48 \\
\hline $\begin{array}{c}\text { Levantar } \\
\text { veda }\end{array}$ & 0,53 & 1 & 0 & 0,27 \\
\hline $\begin{array}{c}\text { Conservaciō } \\
\text { n del acuífero }\end{array}$ & 2,48 & 5 & 0 & 0,50 \\
\hline
\end{tabular}

Fuente: Elaboración propia.

En la tabla 2 se observa la descripción de las variables utilizadas en el modelo. Los entrevistados fueron mayoritariamente del sexo masculino, con un promedio de 44,63 años, tienen una residencia habitual en el distrito, un promedio de 4,63 integrantes por familia, un nivel de educación secundaria a universitaria, y un ingreso promedio de 1640,24 soles, el $92 \%$ está de acuerdo con contar con el servicio de agua potable y el $53 \%$ de los entrevistados opina que se debe levantar la veda por el servicio de agua potable y podría pagar un promedio de 2,48 soles mensuales para conservar el acuífero.

Tabla 3. Proporción de Respuestas Positivas a los Vectores de pago (BID) de la población beneficiaria por el servicio de agua potable en el distrito La Yarada-Los Palos. Tacna 2016.

\begin{tabular}{|c|c|c|c|c|}
\hline BID & \multicolumn{3}{|c|}{ Respuestas } & Proporción \\
\hline S/. & Positivo & Negativo & Total & $\begin{array}{c}\text { Respuesta } \\
\text { positiva }\end{array}$ \\
\hline 10 & 7 & 3 & 10 & 0,70 \\
\hline 20 & 10 & 2 & 12 & 0,83 \\
\hline 30 & 6 & 4 & 10 & 0,60 \\
\hline 40 & 1 & 7 & 8 & 0,13 \\
\hline 50 & 1 & 7 & 8 & 0,12 \\
\hline
\end{tabular}

En la tabla 3, se observa los vectores de pago (BID) mencionados por los encuestados ante el servicio de agua potable en La Yarada-Los Palos. Se muestra un rango de valores de $10 ; 20 ; 30 ; 40$ y 50 nuevos soles, 
siendo el rango de 20 soles el que arroja una mayor proporción de respuestas positivas de 0,83 , seguido del valor de 10 soles con una proporción de respuestas positivas de 0,70 .

Tabla 4. Coeficientes de todas las variables del modelo logístico en la población beneficiaria por el servicio de agua potable en el distrito La Yarada-Los Palos. Tacna 2016.

\begin{tabular}{|c|c|c|c|c|}
\hline Variables & Coeficiente & $\begin{array}{c}\text { Desviación } \\
\text { típica }\end{array}$ & $\mathbf{Z}$ & Valor p \\
\hline Constante & 1,66 & 2,99 & $-0,55$ & 0,58 \\
\hline BID & $-0,09$ & 0,04 & $-2,56$ & 0,01 \\
\hline Sexo & $-0,14$ & 0,83 & $-0,17$ & 0,86 \\
\hline Edad & 0,01 & 0,04 & 0,28 & 0,78 \\
\hline Integrantes & 0,20 & 0,17 & 1,16 & 0,25 \\
\hline $\begin{array}{c}\text { Nivel de } \\
\text { educación }\end{array}$ & 0,66 & 0,52 & 1,27 & 0,20 \\
\hline Ingreso & 0,00 & 0,00 & 0,15 & 0,88 \\
\hline $\begin{array}{c}\text { Acuífero en } \\
\text { veda }\end{array}$ & 1,37 & 1,12 & 1,22 & 0,22 \\
\hline $\begin{array}{c}\text { Levantar } \\
\text { veda }\end{array}$ & 0,34 & 1,01 & 0,33 & 0,74 \\
\hline
\end{tabular}

$\mathrm{DAP}=17,15$ soles

Tamaño de muestra:

Grados de libertad

8

R2 Mc Fadden

0,30

Chi Cuadrado

$19,74^{*}$

Predicción Correcta (\%)

$78,87 \%$

Fuente: Elaboración propia.

En la tabla 4, para contrastar si son o no estadísticamente relevantes las variables que se ha considerado como explicativos de la variable dependiente, se observa que la variable BID (Vectores de Pago) es significativa, rechazando la hipótesis nula a1 $5 \%$, es decir, el coeficiente asociado a la variable BID sea igual a cero; mientras que las otras variables no son significativas, varían entre $20,08 \%$ y $88,82 \%$.

\section{Contraste de significación conjunta}

Asimismo, la prueba de Chi Cuadrado indica que es significativa al 5\% lo que permite rechazar la hipótesis nula existiendo al menos una variable en el modelo diferente a cero, lo cual indica que es un modelo con una predicción del 78,77 \%. El seudo $\mathrm{R}^{2}$ Mc Fadden es de 0,30: esto indica un modelo consistente.

Asimismo, el DAP fue de 17,15 soles para el modelo.

Tabla 5. Proporción de Respuestas Positivas a los BID por el servicio de agua potable en la población beneficiaria y no beneficiaria. Tacna 2016.

\begin{tabular}{|c|c|c|c|c|}
\hline BID & \multicolumn{3}{|c|}{ Respuestas } & Proporción \\
\hline S/. & Positivo & Negativo & Total & $\begin{array}{c}\text { Respuesta } \\
\text { positiva }\end{array}$ \\
\hline 10 & 11 & 7 & 18 & 0,61 \\
\hline 20 & 16 & 4 & 20 & 0,80 \\
\hline 30 & 9 & 7 & 16 & 0,56 \\
\hline 40 & 9 & 13 & 22 & 0,40 \\
\hline 50 & 4 & 10 & 8 & 0,28 \\
\hline
\end{tabular}

Fuente: Elaboración propia.
En la tabla 5 se presenta los vectores de pago (BID) mencionados por los encuestados ante el servicio de agua potable en La Yarada-Los Palos; los cuales muestran un rango de valores de $10 ; 20 ; 30 ; 40$ y 50 nuevos soles, siendo el rango de 20 soles el que arroja una mayor proporción de respuestas positivas de 0,80 seguido del valor de 10 soles con una proporción de respuestas positivas de 0,61 .

Tabla 6. Coeficientes de todas las variables del modelo logístico por el servicio de agua potable en la población beneficiaria y población no beneficiaria. Tacna 2016.

\begin{tabular}{|c|c|c|c|c|}
\hline Variables & Coeficiente & $\begin{array}{l}\text { Desviación } \\
\text { tipica }\end{array}$ & Z & Valor $p$ \\
\hline Constante & 2,46 & 2,05 & 1,20 & 0,23 \\
\hline BID & $-0,05$ & 0,02 & $-2,30$ & $0,02^{\star}$ \\
\hline Sexo & $-0,35$ & 0,62 & $-0,56$ & 0,57 \\
\hline Edad & $-0,02$ & 0,02 & $-1,16$ & 0,24 \\
\hline $\begin{array}{l}\text { Zona de } \\
\text { residencia }\end{array}$ & $-0,63$ & 0,53 & $-1,20$ & 0,23 \\
\hline $\begin{array}{c}\text { Integrantes } \\
\text { de familia }\end{array}$ & 0,03 & 0,16 & 0,23 & 0,81 \\
\hline $\begin{array}{l}\text { Nivel de } \\
\text { educación }\end{array}$ & $-0,33$ & 0,37 & 0,88 & 0,38 \\
\hline $\begin{array}{l}\text { Hectáreas en } \\
\text { producción }\end{array}$ & $-0,01$ & 0,15 & $-0,10$ & 0,92 \\
\hline $\begin{array}{l}\text { Hectáreas } \\
\text { bajo riego }\end{array}$ & 0,26 & 0,38 & 0,67 & 0,50 \\
\hline Ingreso & $-0,79$ & 1,00 & $-0,79$ & 0,43 \\
\hline $\begin{array}{c}\text { Acuítero en } \\
\text { veda }\end{array}$ & $-0,08$ & 0,74 & $-0,11$ & 0,91 \\
\hline $\begin{array}{c}\text { Acces o agua } \\
\text { potable }\end{array}$ & 0,12 & 1,32 & 0,09 & 0,93 \\
\hline $\begin{array}{l}\text { Levantar } \\
\text { veda }\end{array}$ & 0,05 & 0,63 & 0,07 & 0,94 \\
\hline $\begin{array}{c}\text { Pago } \\
\text { adicional }\end{array}$ & 0,70 & 0,20 & 3,47 & $0,00^{* *}$ \\
\hline
\end{tabular}

$\mathrm{DAP}=48.39$ soles

Tamaño de muestra:

Grados de libertad

R2 Mc Fadden

Chi Cuadrado

Predicción Correcta (\%)

Fuente: Elaboración propia.
13

0,28

$33,41 * *$

$75,00 \%$
En la tabla 6, para contrastar si son o no estadísticamente relevantes las variables que se ha considerado como explicativos de la variable dependiente, se observa que la variable BID (Vectores de Pago) es significativa rechazando la hipótesis nula al $5 \%$, es decir, el coeficiente asociado a la variable BID sea igual a cero. Asimismo, la variable pago adicional para la conservación del acuífero es significativo al $1 \%$, mientras que las otras variables no son significativas variando entre $22,87 \%$ y $94,27 \%$.

\section{Contraste de significación conjunta}

La prueba de Chi Cuadrado indica que las variables estudiadas son significativas al $5 \%$, lo cual significa que se rechaza la hipótesis nula existiendo al menos una variable en el modelo, es diferente a cero. Esto indica que es un modelo con una predicción del 75,00\%. El seudo $\mathrm{R}^{2} \mathrm{Mc}$ Fadden es de 0,28 lo que demuestra que es un modelo consistente. 
Por otro lado, elDAP fue de 48,39 soles estimado por el modelo.

\section{DISCUSIÓN \\ Encuesta abierta por el servicio de agua potable en la población beneficiaria}

El análisis de los resultados de la encuesta abierta reveló que el $96,88 \%$ de los pobladores considera que es importante la conservación del acuífero valle Caplina para el desarrollo de sus actividades agrícolas y económicas. Asimismo, solamente el $48 \%$ de los agricultores sabe que el acuífero se encuentra en veda, el $79,2 \%$ considera que para usar las aguas en $\mathrm{La}$ Yarada-Los Palos necesita de licencia y que están bajo amenaza por la sobreexplotación indiscriminada, por tanto deberían protegerse. Asimismo, el $72,2 \%$ manifiesta que se debería levantar la veda para contar con el servicio de agua potable. Es importante resaltar que el acuífero del valle Caplina se encuentra en veda desde 1980, por contar con una limitada recarga como indica la Autoridad Nacional del Agua (2013); actualmente el acuífero está siendo sobreexplotado y podría colapsar perjudicando a los agricultores formalmente establecidos, como sucede en los acuíferos de México reportado por Aragón et al. (2015), quienes manifiestan que la sobreexplotación modifica las condiciones hidrogeoquímicas de los acuíferos, lo que revela prácticas no sustentables (Soto, 2007).

De igual forma, cuando se les preguntó sobre la importancia de contar con el servicio de agua potable el $96,88 \%$ considera que es necesario contar con este servicio, el $84,4 \%$ manifiesta que utiliza para uso doméstico las aguas de los pozos de uso agrícola, por lo que el $64,48 \%$ está insatisfecho por la calidad del agua y el $82,2 \%$ indica que les causa problemas en la salud: afirmación corroborada con la información proporcionada por el Centro de Salud de La Yarada (2016), y que coincide con las investigaciones de otros países como Argentina, que señalan que ocasiona cáncer a la piel, por la presencia de arsénico en las aguas subterráneas (Bocanegra et al.,2002).

Asimismo, los encuestados indican que estarían dispuestos a pagar mensualmente para contar con el servicio de agua potable, efectuando un pago adicional para conservar el acuífero del valle Caplina y que sigan brindando servicios ambientales a la población, el 75 $\%$ contestó afirmativamente. $\mathrm{Al}$ respecto Barrantes y Flores (2013), en investigaciones similares revelan que en Pasco- Perú, se podría recaudar anualmente un aproximado de 1,95 millones de soles para la implementación del Programa de Conservación y Mejoramiento de Pastizales (PCMP). Asimismo, Brunett et al. (2010) mencionan que estarían dispuestos a pagar por los servicios ambientales para la conservación del acuífero del Parque Nacional Nevado de Toluca (México).

\section{Vectores de pago (BID) por el servicio de agua potable en la población beneficiaria}

Cuando se les preguntó sobre su disposición a pagar por un determinado BID mensual por el servicio de agua potable, la respuesta variaba entre 10 a 50 soles, sugiriendo que los vectores de pago (BID) más adecuados para definir la función de pago serían de 10; $20 ; 30 ; 40$ y 50 soles. Al respecto, se pueden considerar 5 valores o 6 valores (INA-CELA, 2016; Oaxaca, 1997). Se encontró que la proporción de respuestas positivas disminuía cuando el vector de pago aumentaba (tabla 3), aspecto consistente con la teoría económica de la demanda por un bien o servicio; es decir, a menor vector de pago mayor la disposición a pagar, como lo encontrado por Barrantes y Flores (2013) en investigaciones similares. También AvilésPolanco et al. (2010) refieren que existe una mayor disposición a pagar de la población beneficiaria por el servicio de agua potable, pero conservando el acuífero.

\section{Valor de uso del agua en la población beneficiaria}

Los resultados de la primera corrida del programa solamente con los agricultores que usan las aguas del acuífero Caplina (valor de uso), en encuesta cerrada a los pobladores de La Yarada-Los Palos con todas las variables en términos de valor de los coeficientes, desviación típica y significancia estadística se muestran en la tabla 2 , observándose que el coeficiente de regresión de la BID para el modelo LOGIT fue negativo $(p<0,01)$ mientras que el coeficiente para el ingreso mensual fue positivo, pero no significativo $(p>0,05)$. Lo cual confirma que a mayor valor de un bien la disponibilidad a pagar por el mismo es menor, en tanto que a mayor ingreso familiar la tendencia es a pagar más por el mismo. Analizando el comportamiento de las otras variables se encuentra que los beneficiarios valoran el acuífero valle Caplina en $34 \%$ y consideran que se debe levantar la veda para contar con el servicio de agua potable porque el acuífero constituye la fuente de la actividad económica y si el encuestado tiene más años de estudio, la DAP aumenta; mientras que en aquellos que son de familia numerosa, la DAP disminuye. En referencia a la bondad de ajuste del modelo, los valores de la $\mathrm{R}^{2} \mathrm{Mc}$ Fadden $\left(\mathrm{R}^{2}=0,30\right)$ para una DAP de $\mathrm{S} / 17,15$, basada en variables socioeconómicas, se observó que este fue superior a los encontrados en similares estudios $\left(R^{2} \mathrm{Mc}\right.$ Fadden $<0,0779$, Loomis et al., 2008) y otros en que se utilizó Chi cuadrado como indicador de la relación significativa entre el BID y la proporción de familias dispuestas a pagar por determinado BID, Villena \& Lafuente (2012). Es necesario precisar que en otros países latinoamericanos la tendencia es pagar para la conservación de las fuentes naturales que proveen del recurso hídrico a las ciudades (Bockor et al., 2005). 
Valor de uso y no uso del agua en la población beneficiaria y población no beneficiaria

En la segunda corrida se analizó con la población total (población que usa las aguas subterráneas directamente y población que no utiliza las aguas directamente) todas las variables en términos de valor de los coeficientes, desviación típica y significancia estadística. Estos datos se muestran en la tabla 6, observándose que el coeficiente de regresión de la BID para el modelo LOGIT fue negativo $(p<0,01)$ mientras que el coeficiente para el ingreso mensual fue positivo, pero no significativo ( $p>0,05)$. Lo cual confirma que cuanto mayor sea el valor de un bien, la disponibilidad a pagar por el mismo es menor; en tanto que a mayor ingreso familiar, la tendencia es a pagar más por el mismo. Analizando el comportamiento de las otras variables se encuentra que los agricultores valoran el acuífero del valle Caplina y el $8 \%$ considera que se debe levantar la veda para contar con el servicio de agua potable, ya que el acuífero constituye la fuente de la actividad económica y además porque es considerada un acuífero de reserva para la población de Tacna, de un gran valor y legado para las generaciones futuras. En referencia a la bondad de ajuste del modelo, los valores de la $\mathrm{R}^{2} \mathrm{Mc}$ Fadden $\left(\mathrm{R}^{2}=0,28\right)$ indican un modelo consistente para una DAP de $\mathrm{S} / 48,39$ por el servicio de agua potable, un valor económico alto a considerar cuando se toma decisiones en la gestión del agua potable de La Yarada-Los Palos, además del costo adicional mensual en el recibo de agua potable, de 2,48 soles, por la conservación del acuífero del valle Caplina. En relación con esto, como mencionamos anteriormente, existe la tendencia en Latinoamérica a pagar por los servicios ambientales que brindan los bosques, la reserva nacional y los acuíferos, tal como indica Martínez y Dimas (2007); además refieren que la DAP de los entrevistados fue de US $\$ 3,46$ familia/mes, y la suma de las disposiciones a pagar de los habitantes de un total de US\$132 mil/año.

\section{CONCLUSIONES}

Empleando el método de regresión logística, modelo Logit elegido por ser confiable y real, se arribó a las siguientes conclusiones:

El valor económico de uso por el servicio de agua potable mensual referido por la población beneficiaria encuestada es de 17,15 soles. Asimismo, el valor económico total (valor de uso y no uso) por el servicio de agua potable señalado por la población beneficiaria y no beneficiaria es de 48,39 soles. También el pago adicional mensual señalado por la población beneficiaria y no beneficiaria para la conservación del acuífero del valle Caplina es de 2,48 soles, porque valoran este recurso para las futuras generaciones.

Por último, las variables sociales y ambientales estudiadas no fueron significativas en la población encuestada.

\section{REFERENCIAS BIBLIOGRÁFICAS}

Adkins, L.C.(2014). Using gretl for Principles of Econometrics, 4th Edition Version 1.0411. Recuperado de: http:/www.learneconometrics.com/gretl/usin g_gretl_for_POE4.pdf

Aragón, M., Escolero, O., Navarro, S., y Ortiz, M. (2015). Distribución geográfica de arsénico en acuífero de los Valles Centrales de Oaxaca, México. Ingeniería hidráulica y ambiental, 36, (1),102-110. Recuperado de: http://scielo.sld.cu/pdf/riha/v36n1/riha08115. pdf.

Autoridad Nacional del Agua (2013). Acuífero Caplina. Recuperado de:

http://www2.congreso.gob.pe/sicr/comisiones /2012/com2012ciencia.nsf/0/42fcb88a2960f0 4c05257b5f00783c2d/\$FILE/10 Acuifero C aplina Tacna.pdf.

Autoridad Nacional del Agua (2013). Situación actual y perspectivas en el sector agua y saneamiento en el Perú. Seminario de "Tecnología alemana en el rubro de Agua y Saneamiento". Recuperado de : http://www.camaraalemana.org.pe/downloads/2-130311-ana.pdf.

Autoridad Nacional del Agua (2014). Informe Técnico del Primer Monitoreo Participativo de Calidad de Agua Subterránea del acuífero Caplina. Realizado del 23 de junio 2014 al 27 junio 2014. Proyecto de Modernización de la Gestión de los Recursos Hídricos. Tacna.

Avilés-Polanco, G, Leonardo Huato, L., TroyoDiéguez, E. Murillo, B., García, J.L Beltrán, L.F (2010). Valoración económica del servicio hidrológico del acuífero de La Paz, B.C.S.: Una valoración contingente del uso de agua municipal. Frontera Norte, 22(43).

Barrantes, C y Flores, E. (2013). Estimando la Disposición a Pagar por la conservación de los pastizales alto andinos. Departamento Académico de Biología, Universidad Nacional Agraria La Molina, Lima - Perú. Ecología Aplicada, 12(2).

Bocanegra, O, Bocanegra, E. y Alvarez, A. (2002). Arsénico en aguas subterráneas: Su impacto en la salud. Recuperado de:

http://www.cofes.org.ar/descargas/info sector Arsenico/Bocanegra2 Alvarez pdAs Estudi o Bocanegra.pdf.

Bockor, I., Escobedo, M., Sales, E., Ovando, M. (2005). Valoración del agua como servicio ambiental para el abastecimiento de agua potable en el casco municipal de San Jerónimo, Baja Verapaz. Guatemala. Cooperación Técnica Alemana Programa para la Descentralización y Desarrollo Municipal Gestión Ambiental y de Riesgo Componente implementado por: DDM/GTZ/ GFA Consulting Group. 
Brunett, E; Baró, J. E.; Cadena, E; Esteller, M. V. (2010). Pago por servicios ambientales hidrológicos: caso de estudio Parque Nacional del Nevado de Toluca, México. Ciencia Ergo Sum, 17(3) 286-294.

Centro de Salud de la Yarada (2016). Información Estadística.

Chaves, E. (2008). Valoración del agua en la cuenca del río Tempisque: Un ejemplo sobre el método de valoración contingente. UNICIENCIA, 22, 19-31.

Fasciolo, G.(2002). Valoración contingente. El Análisis de Datos en el Enfoque de la Respuesta Dicotómica. Mendoza. INACELA. Instituto Nacional del Agua. Recuperado de:

https://econamunsa.files.wordpress.com/ 2012/04/mvc analisis de datos fasciolo 2002.pdf.

Fasciolo, G. y Mendoza, V. (2002). El método de la valoración contingente. Apuntes inéditos. INA. CELA.

Moscote, O. y Arley, W. (2012). Modelo Logity Probit in caso de aplicación. Recuperado de: https://www.google.com.pe/search?q=mo delo+logit+y+probit+revistas+universida d\&rlz=1C1HLDY_esPE742PE742\&oq= modelo+logit+y+probit+revistas+univers idad\&aqs=chrome..69i57.33599j0j9\&sou rceid $=$ chrome $\&$ ie $=$ UTF- 8

Oaxaca, J. (1997). Estimación de la Disposición a Pagar por Abasto de Agua para el Área Metropolitana de Monterrey. (Tesis de maestría). Universidad Autónoma de Nuevo León. Recuperado de:

file:///E:/valoracion $\% 20 \mathrm{del} \% 20 \mathrm{agua} / \mathrm{TES}$ IS\%20DEFINITIVO\%202017/GLEN/10 20120076.PDF.

Riera, et al. (2005). Manual de Economia Ambiental y de los Recursos Naturales. Thomson Editores Spain.

Soto, G. M. de O (2007). Tarifas, escasez $y$ sustentabilidad en las mega ciudades. ¿Cuánto están dispuestos a pagar los habitantes de la ciudad de México? México: Universidad Iberoamericana, Procuraduría Ambiental y del Ordenamiento Territorial, Sistema de Aguas de la Ciudad de México y Centro de Estudios Jurídicos y Ambientales.

Villena, M \& Lafuente, E. (2012). Valoración económica de bienes ambientales por beneficiarios circundantes y no circundantes. Cuadernos de Economía $31,(56), 33$.

Whitehead, J. (1990). Measuring willingness-to-pay for wetlands preservation with the contingent valuation method. Wetlands, 10(2), 187-201. 